\section{(2)} OPEN ACCESS

\title{
Role of advanced left ventricular imaging in adults with aortic stenosis
}

\author{
Andreea Calin, ${ }^{1,2}$ Anca D Mateescu, ${ }^{1,2}$ Andreea C Popescu, ${ }^{1,3}$ Rong Bing, ${ }^{4}$ \\ Marc R Dweck, ${ }^{4}$ Bogdan A Popescu (D) ${ }^{1,2}$
}

- Additional material is published online only. To view please visit the journal online (http://dx.doi.org/10.1136/ heartjnl-2019-315211).

${ }^{1}$ Department of Cardiology, Euroecolab, University of Medicine and Pharmacy "Carol Davila", Bucharest, Romania 2Department of Cardiology, Emergency Institute for Cardiovascular Diseases "Prof Dr C C lliescu", Bucharest, Romania

${ }^{3}$ Department of Cardiology, Emergency University Hospital Elias, Bucharest, Romania ${ }^{4}$ BHF Centre for Cardiovascular Science, The University of Edinburgh, Edinburgh, EH16 4SB, United Kingdom

\section{Correspondence to}

Professor Bogdan A Popescu, Department of Cardiology, University of Medicine and Pharmacy "Carol Davila" - Euroecolab, Emergency Institute for Cardiovascular Diseases "Prof Dr C C Iliescu", Sos. Fundeni 258, 022328 Bucharest, Romania; bogdan.a.popescu@gmail.com

Received 20 November 2019 Revised 17 January 2020 Accepted 17 February 2020 Published Online First 16 March 2020

\section{Check for updates}

(C) Author(s) (or their employer(s)) 2020. Re-use permitted under CC BY-NC. No commercial re-use. See rights and permissions. Published by BMJ.

\begin{tabular}{l}
\hline To cite: Calin A, \\
Mateescu AD, \\
Popescu AC, et al. Heart \\
2020;106:962-969. \\
\hline
\end{tabular}

\section{ABSTRACT}

This review focuses on the available data regarding the utility of advanced left ventricular (LV) imaging in aortic stenosis (AS) and its potential impact for optimising the timing of aortic valve replacement. Ejection fraction is currently the only LV parameter recommended to guide intervention in AS. The cut-off value of $50 \%$, recommended for decision-making in asymptomatic patients with AS, is currently under debate. Several imaging parameters have emerged as predictors of disease progression and clinical outcomes in this setting. Global longitudinal LV strain by speckle tracking echocardiography is useful for risk stratification of asymptomatic patients with severe AS and preserved LV ejection fraction. Its prognostic value was demonstrated in these patients, but further work is required to define the best thresholds to aid the decision-making process. The assessment of myocardial fibrosis is the most studied application of cardiac magnetic resonance in AS. The detection of replacement fibrosis by late gadolinium enhancement offers incremental prognostic information in these patients. Clinical implementation of this technique to optimise the timing of aortic valve intervention in asymptomatic patients is currently tested in a randomised trial. The use of T1 mapping techniques can provide an assessment of interstitial myocardial fibrosis and represents an expanding field of interest. However, convincing data in patients with AS is still lacking. All these imaging parameters have substantial potential to influence the management decision in patients with AS in the future, but data from randomised clinical trials are awaited to define their utility in daily practice.

\section{INTRODUCTION}

Aortic stenosis (AS) is the most common valvular heart disease in adults, with an increasing prevalence in the ageing population. ${ }^{1}$ Calcific AS is a complex disease that includes changes in the valve and myocardium. ${ }^{2}$ The response of the left ventricle (LV) to pressure overload in AS ultimately contributes to symptom occurrence, and its consequences impact management and outcomes.

Over the last decade, research related to LV structure and function in patients with AS has increased, due to improvements in imaging modalities and potential therapies, in particular the emergence of transcatheter aortic valve implantation (TAVI). This has prompted focus on subclinical changes in LV function, as well as the degree of reversibility of LV structural changes in advanced stages of ASfactors which may influence the optimal timing of valve intervention.
In this review, we summarise the available data on the role of advanced LV imaging in AS, focusing on the pathophysiological insights that they provide and their potential impact on clinical decision-making.

\section{LV response to AS}

In patients with significant AS, pressure overload causes an increase in LV wall stress that stimulates myocyte enlargement and increases in LV wall thickness. Concentric hypertrophy is the primary compensatory mechanism that leads to increased contractile force and reduced systolic wall stress. ${ }^{3}$

The magnitude of LV hypertrophy (LVH) is poorly linked to AS severity ${ }^{4}$ suggesting that other determinants are also involved in its development. Age, gender, angiotensin-converting enzyme I/D polymorphism, co-existing coronary artery disease and hypertension are additional factors influencing the LV response to AS. ${ }^{5}$ Reduced systemic arterial compliance independently contributes to increased afterload and may influence LV remodelling. ${ }^{7}$

Women exhibit different patterns of LV remodelling, less LVH, and a lower degree of focal fibrosis and extracellular expansion compared with men with similar AS severity, age and functional status. ${ }^{8}$

Although beneficial initially, ultimately the hypertrophic response can decompensate, with patients transitioning to heart failure, symptoms and adverse events (figure 1). Myocyte degeneration, cell death and fibrosis have been described as the key structural changes responsible for this transition. ${ }^{9}$ Increased myocardial oxygen demand, unbalanced by an insufficient increase in the coronary capillary network, is thought to lead to impaired coronary flow reserve and myocardial perfusion, with increased cardiomyocyte cell death. ${ }^{10}$ Moreover, myocardial supply can also be reduced as a result of decreased coronary perfusion pressure, due to the presence of AS. ${ }^{11}$

Histopathological studies have shown that myocardial fibrosis in particular is an integral part of myocardial disease progression in AS. ${ }^{12}$ The mechanisms governing the development and progression of myocardial fibrosis are incompletely understood. An imbalance in matrix metalloproteinases and tissue inhibitors of matrix metalloproteinase activity, alongside increases in angiotensin-converting enzyme and transforming growth factor beta1 activity contribute to the development of fibrosis, ${ }^{13} 14$ and each of these mechanisms could be a potential target for interventions. Myocardial fibrosis has traditionally been categorised into diffuse interstitial fibrosis 

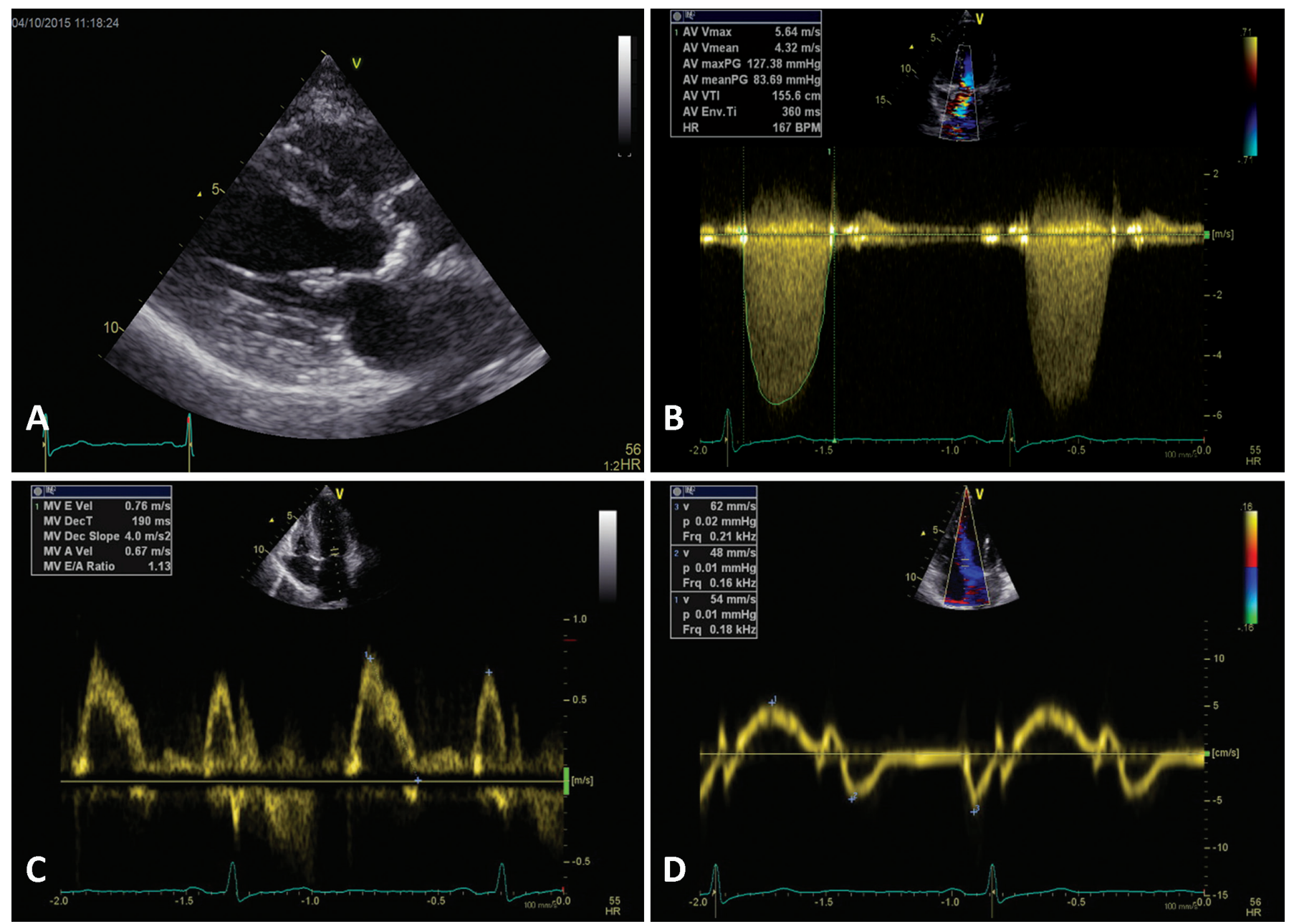

Figure 1 Consequences of very severe calcific aortic stenosis $(A)$ in a patient in their 60 s without a history of systemic hypertension. Peak transvalvular gradient was $127 \mathrm{~mm} \mathrm{Hg}$ (mean gradient of $84 \mathrm{~mm} \mathrm{Hg}$ ) (B) at a blood pressure of 120/70 mm Hg, with an estimated LV systolic pressure of $247 \mathrm{~mm} \mathrm{Hg}$ and a calculated aortic valve area of $0.6 \mathrm{~cm}^{2}$. As a result of severe pressure overload, there was significant concentric LV hypertrophy (A) with an indexed LV mass of $130 \mathrm{~g} / \mathrm{m}^{2}$ and a relative wall thickness of 0.55 . Global systolic LV function was preserved (ejection fraction of $65 \%$ ) while systolic myocardial velocities measured by tissue Doppler imaging were significantly reduced (septal s' $5.2 \mathrm{~cm} / \mathrm{s}$ )(D), indicating LV longitudinal dysfunction. There was significant LV diastolic dysfunction: impaired relaxation with a septal e' of $4.8 \mathrm{~cm} / \mathrm{s}$ (D) and increased filling pressurepseudonormal mitral inflow $(\mathrm{C})$ with elevated E/e' ratio of 16 and a moderately dilated left atrium. LV, left ventricular.

and replacement fibrosis. Interstitial fibrosis occurs earlier in the disease process and appears to be reversible with afterload relief. Replacement fibrosis is the result of myocyte necrosis and replacement of these cells with extracellular matrix. This typically occurs in a non-infarct distribution and is largely irreversible. However, recent elegant histological work utilising myocardial biopsies and cardiac magnetic resonance (CMR) has shed further light on the complexities of myocardial fibrosis in AS, demonstrating frequent overlap in these forms of fibrosis and that a gradient exists in myocardial fibrosis extending from the sub-endocardium to the mid-myocardium. ${ }^{15}$

\section{Echocardiography in the assessment of the LV in AS}

Echocardiography is the first-line imaging modality to evaluate patients with AS. An accurate and precise assessment of both LV remodelling and function is warranted in all patients with AS. Moreover, echocardiography allows assessment of structural and functional changes in the atria and the right ventricle, with a stepwise increase in mortality observed with progressive cardiac damage. ${ }^{2}$

\section{LV remodelling}

Linear LV dimensions must be measured to estimate LV mass and relative wall thickness, for LV remodelling classification. ${ }^{16} \mathrm{LVH}$ is estimated based on conventional two-dimensional (2D) echocardiography measurements of wall thickness in the septum and inferolateral wall. ${ }^{16}$ The three-dimensional (3D) echocardiography assessment of LV mass, validated against CMR, overcomes the geometrical assumptions inherent in 2D echocardiography, with improved accuracy. ${ }^{17}$ However, the lack of specific cut-off values currently hampers its widespread clinical use.

\section{LV diastolic function}

LV diastolic dysfunction is one of the earliest consequences of LVH and myocardial fibrosis in AS. Although LV diastolic function is a sensitive marker of myocardial changes, it lacks specificity and does not play a role in timing intervention for patients with AS. LV diastolic dysfunction is associated with increased mortality, worsens with progressive myocardial remodelling before aortic valve replacement (AVR), and gradually improves with reverse remodelling after AVR. ${ }^{18}$ The improvement of LV 
diastolic dysfunction in AS takes longer than the reversal of LV systolic dysfunction since the former is mainly related to longstanding LV structural changes while the latter also reflects afterload mismatch. ${ }^{19} 20$

\section{LV systolic function}

The assessment of LV systolic performance begins with measuring LV ejection fraction (LVEF). ${ }^{21}$ The recommended 2D echocardiography method is the biplane method of discs. ${ }^{16}$ Whenever feasible, the measurement of LVEF by $3 \mathrm{D}$ echocardiography is recommended. ${ }^{16}$

Although LVEF carries important prognostic information and guides therapy, it is load-dependent and not an index of myocardial contractility. LVEF is also often confounded in severe AS by the presence of $\mathrm{LVH}^{22}$ and remains in the normal range until late in the disease course, even when myocardial fibrosis and longitudinal dysfunction are already present. $^{23}$

Assessment of LV deformation detects earlier changes in myocardial function and allows a better understanding of progression to heart failure in AS. ${ }^{24-26}$ Systolic longitudinal strain parameters, assessed by tissue Doppler imaging (TDI), are significantly decreased in patients with AS and preserved LVEF and their decline is related to AS severity. ${ }^{25}$ These parameters improve rapidly after AVR, preceding any substantial changes in LV mass and EF, and suggesting their partial dependence on LV afterload. $^{2526}$

However, the assessment of LV deformation by TDI requires parallel alignment of the Doppler beam with myocardial motion direction and cannot be performed for all the LV segments.

Speckle-tracking echocardiography (STE) overcomes these limitations, allowing a multidirectional evaluation of myocardial deformation. ${ }^{27}$ In AS, LV longitudinal strain is impaired, especially in the basal segments, and is a predictor of clinical events in asymptomatic AS (figure 2). ${ }^{24} 28$ The primary mechanism involved in the alteration of LV longitudinal strain in AS is the development of LV fibrosis: both related to the valve disease and prior myocardial infarction. ${ }^{23}$ Moreover, global longitudinal strain (GLS) also depends on the pattern of LV remodelling, with lower values in patients with significant concentric LVH. ${ }^{29}$ An increasing body of evidence suggests the usefulness of GLS for risk stratification and management of asymptomatic patients with severe AS and preserved LVEF. ${ }^{24}$ Recent studies have shown that GLS predicts postoperative LV dysfunction and outcomes better than $\mathrm{EF}^{30}$ GLS improves early after AVR, ${ }^{31}$ reflecting its relationship to afterload mismatch as well as fibrosis, although this area requires further study.

A progressive transmural impairment of myocardial function, with a gradual decrease in circumferential and radial deformation, was demonstrated with increasing afterload in AS. ${ }^{32}$

Mechanical dispersion measured by STE reflects LV mechanical dyssynchrony and has been linked to myocardial fibrosis and ventricular arrhythmias in myocardial infarction and cardiomyopathies. ${ }^{33}{ }^{34}$ Increased LV mechanical dispersion is associated with adverse outcome in patients with AS, independently of LVEF, flow status or treatment. ${ }^{35}$ Mechanical dispersion appears to be less afterload dependent than LVEF and GLS, and may provide novel prognostic information in AS (figure 3).

\section{CMR imaging}

The role of CMR has burgeoned in recent years, largely due to advances in scanner technology, software and accessibility. CMR offers the distinct advantage of combining multiparametric assessments of anatomy, function with detailed soft tissue characterisation. Indeed, its ability to identify, quantify and discriminate between myocardial diseases is superior to other currently available imaging modalities. This is of importance in AS, where an understanding of myocardial health and function may be of clinical relevance, beyond standard measures of valve haemodynamics and obstruction. Currently, the use of CMR to detect myocardial fibrosis is the most studied application of myocardial tissue characterisation in AS.
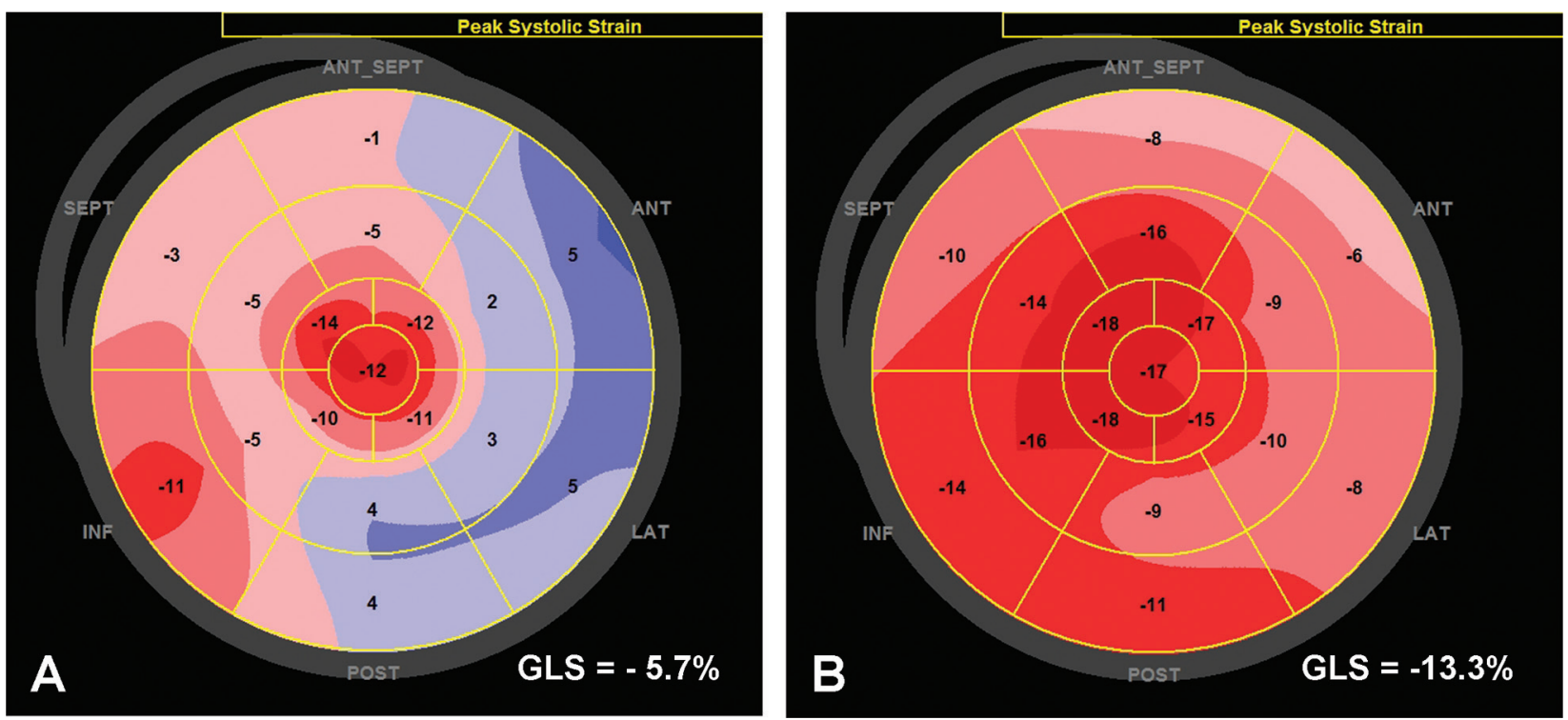

Figure 2 LV global longitudinal strain measured by speckle tracking echocardiography in a patient with severe aortic stenosis before (A) and 1 month after TAVI (B). Before TAVI, the LVEF was 30\% and the mean transvalvular gradient was $70 \mathrm{~mm} \mathrm{Hg}$. The GLS was severely reduced: $-5.7 \%$. Systolic lengthening is present in the basal and mid-segments of the lateral wall. Angiography revealed no significant coronary artery disease. One month after TAVI, LVEF increased to 55\% while GLS significantly improved: $-13.3 \%$. GLS, global longitudinal strain; LV, left ventricle; LVEF, LV ejection fraction; TAVI, transcatheter aortic valve implantation. 

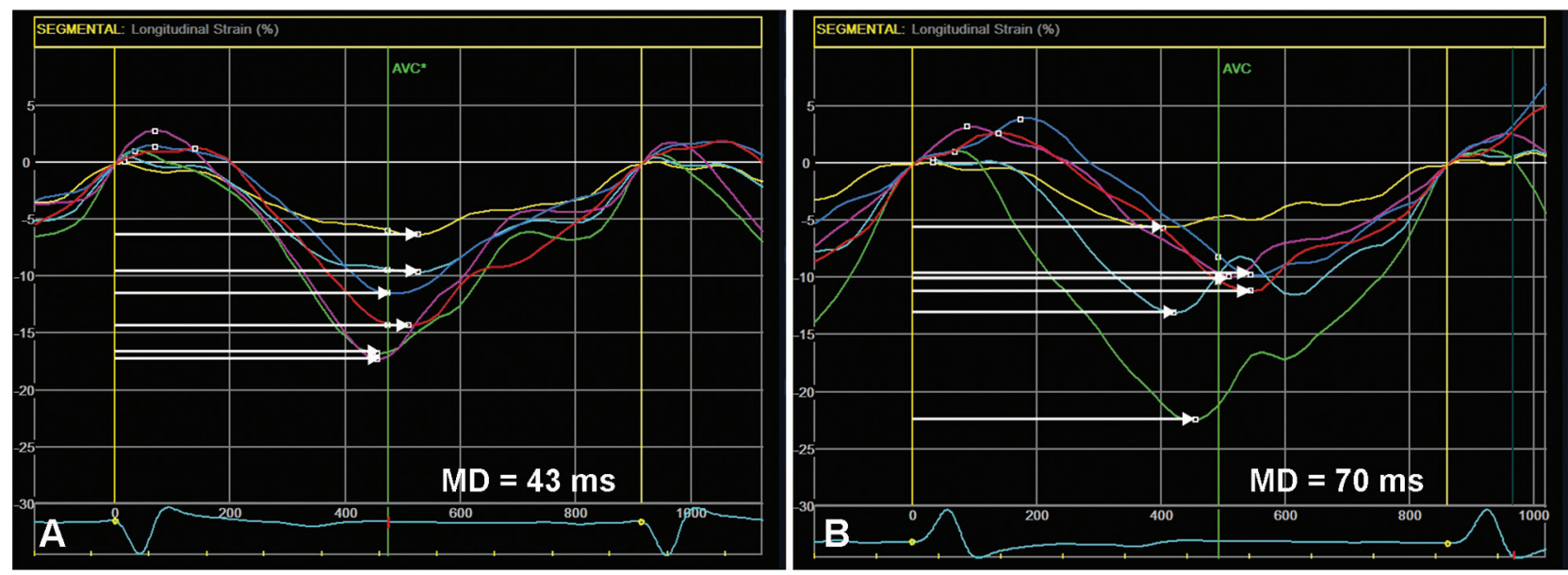

Figure 3 LV mechanical dispersion assessed by speckle-tracking echocardiography in the four-chamber view in two patients with severe aortic stenosis and preserved LV ejection fraction. White horizontal arrows indicate contraction duration, defined as the time from QRS onset on the ECG to peak negative longitudinal strain. Mechanical dispersion is defined as the SD of contraction duration from all LV segments. (A) Low value of mechanical dispersion indicating a homogeneous contraction pattern. (B) Pronounced mechanical dispersion in a patient with significant LV hypertrophy and complex ventricular arrythmia. LV: Left ventricular.

\section{Replacement fibrosis: late gadolinium enhancement}

Replacement fibrosis is detected with CMR using late gadolinium enhancement (LGE). Gadolinium-based contrast agents partition into extracellular space and wash out of areas of focal fibrosis slower than healthy tissue. This provides a clear visual contrast between focal extracellular matrix expansion and healthy myocardium which can also be quantified (figure 4).

LGE has been studied in various myocardial pathologies and is universally associated with an increased risk of adverse outcome. A large body of observational data has demonstrated this same association in AS. ${ }^{46-39}$ Indeed, multiple studies have consistently shown strong independent associations between ischaemic and non-ischaemic LGE and both cardiovascular and all-cause mortality. Furthermore, the development of nonischaemic LGE in AS appears to serve as an objective marker of LV decompensation and portends further rapid progression of fibrosis burden. ${ }^{40}$ Importantly, this fibrosis does not regress after $\mathrm{AVR}^{15}{ }^{40}$ the burden of scarring that develops while awaiting surgery persists in patients for life. This is important because the more myocardial fibrosis, the worse the long-term prognosis. ${ }^{38}$ The detection of LGE in AS may therefore offer incremental prognostic information. Clinical implementation of LGE to optimise the timing of aortic valve intervention is being tested in the randomised Early Valve Replacement Guided by Biomarkers of LV Decompensation in Asymptomatic Patients with Severe AS trial. ${ }^{41}$

\section{Diffuse interstitial fibrosis: T1 mapping}

Unlike LGE, which is insensitive for the detection of diffuse interstitial fibrosis, T1 mapping techniques can provide overall assessments of the extracellular compartment. While providing a close surrogate assessment of myocardial fibrosis, ${ }^{42}$ these markers are also affected by other extracellular factors including oedema and capillary volume. The most studied methods are native T1, which does not require gadolinium contrast, and extracellular volume fraction (ECV\%). ${ }^{43}$ Native T1 provides myocardial T1 times which can vary depending on the type of myocardial pathology present, while ECV\% provides an estimate of the ECV expressed as a fraction of total myocardial volume. These parameters have prognostic significance across a range of cardiomyopathies and have been the focus of substantial research to define normal values and to develop sequences that can be applied across different scanners. The indexed ECV (or matrix volume) has also been studied in AS; this measure provides an estimate of absolute $\mathrm{ECV}(\mathrm{ECV} \times \mathrm{LV}$ myocardial volume $)$ and therefore the total burden of myocardial fibrosis. T1 mapping has provided important insight into the myocardium in AS, most notably the potential for diffuse fibrosis to reverse post-AVR, with an increasing body evidence demonstrating its prognostic power in AS as with other conditions. ${ }^{44}$

The role of different LV imaging parameters in patients with AS is presented in table 1.

\section{Other diagnostic modalities}

Recent data have demonstrated the potential of CT for both tissue characterisation and deformation assessments. Specifically, small studies have shown that CT-derived ECV\% correlates with CMR-derived ECV\% as well as histology, ${ }^{45} 46$ while CT-derived GLS also appears to be feasible in selected patients with good image quality. ${ }^{47}$ Interestingly, CT-derived GLS correlated well with CT-derived ejection fraction, but only modestly with echocardiography-derived GLS. These advances are of particular interest given the routine use of CT for planning TAVI and may improve risk prediction. ${ }^{48}$ Additionally, the detection of a very high ECV\% in this context is potentially important due to the relatively high prevalence of cardiac amyloid in this patient population. ${ }^{49}$ Finally, there is also interest in applying advanced molecular imaging techniques to interrogate myocardial disease activity in addition to the structural and functional assessments provided by current modalities. In particular, positron emission tomography using radiotracers to detect fibrosis activity holds major promise in different disease settings, including AS.

The imaging data must be integrated with clinical data and other information. Strain pattern on 12-lead ECG has a high specificity (but low sensitivity) for LVH and provides prognostic information. ${ }^{50}$ Increased levels of BNP and troponins are associated with LVH, fibrosis and a worse prognosis. ${ }^{\text {w1-w3 }}$ However, these biomarkers are not specific to AS; therefore, a combined imaging-biomarker approach may work best. 

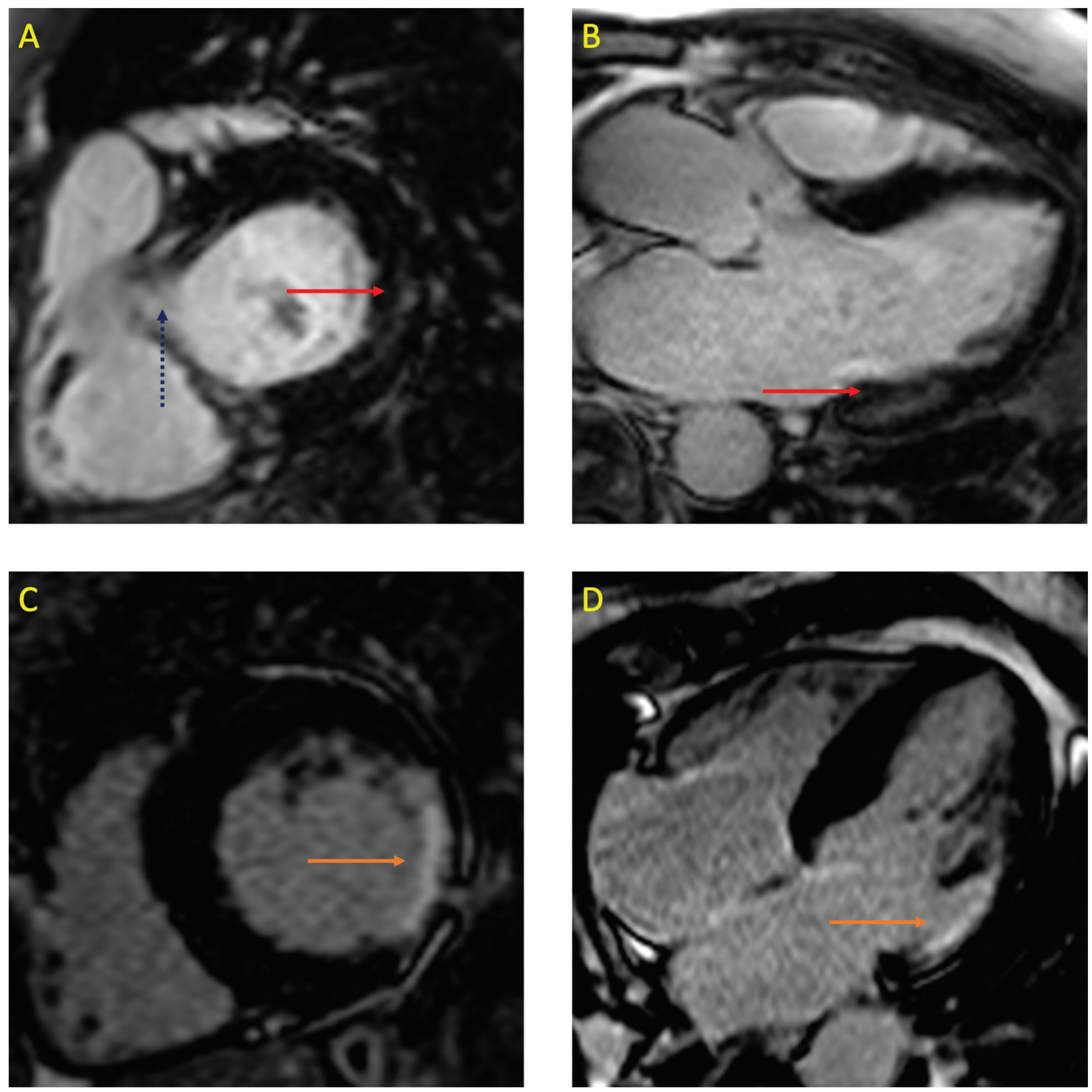

Figure 4 LGE on CMR imaging. Panels A and B are short axis and three-chamber views of a patient with aortic stenosis. The red arrows indicate focal, non-ischaemic (mid-wall) LGE in the basal inferolateral wall. The dotted blue arrow in panel A indicates the left ventricular outflow tract, where caution must be taken in interpreting contrast enhancement due to partial volume effects. in contrast, panels $C$ and $D$ are the same views of a different patient with a myocardial infarction involving the same segments (orange arrows). Note the transmural pattern and wall thinning. LGE, Late gadolinium enhancement; CMR, cardiac magnetic resonance.

\section{Implications of recent imaging advances in different clinical scenarios in AS}

Asymptomatic severe AS

In physically active patients, an exercise test is recommended to reveal symptoms or a fall in blood pressure. ${ }^{21}$ Ejection fraction is the only LV parameter currently recommended to guide intervention in asymptomatic patients with AS, with a cut-off value of $<50 \%$ for referral to AVR. ${ }^{21}$ The usefulness of LVEF in this setting is questioned, as few patients with severe AS and LVEF $<50 \%$ are asymptomatic ${ }^{\mathrm{w} 4}$ and there are no robust outcome data to support this threshold. Furthermore, the cut-off value of $<50 \%$ is problematic since in asymptomatic patients there is evidence that LVEF $<55 \%$ correlates with excess mortality in medically managed patients and is an independent determinant of poorer survival after surgical AVR. ${ }^{\text {w5 }}$

The assessment of GLS could be more appropriate to detect subclinical LV dysfunction in this setting. An individual participant data meta-analysis ${ }^{24}$ including 1067 asymptomatic patients with significant AS and LVEF $>50 \%$, confirmed that GLS is strongly associated with mortality, with $>2.5$ fold increase in risk of death in patients with GLS $<14.7 \%$ while patients with GLS $>18 \%$ had an excellent outcome. These data support the systematic measurement of GLS in asymptomatic patients with AS and preserved LVEF and may promote its use in clinical practice. Future trials to establish clear thresholds and incorporating GLS into decision-making for asymptomatic patients with AS 
Table 1 Role of imaging techniques in assessing the left ventricle in patients with aortic stenosis

\begin{tabular}{|c|c|c|}
\hline & Advantages & Disadvantages \\
\hline \multicolumn{3}{|l|}{ Echocardiography } \\
\hline $\begin{array}{l}\text { LV mass and } \\
\text { RWT }\end{array}$ & $\begin{array}{l}\text { Easy to access } \\
\text { Useful for classification of LV } \\
\text { remodelling } \\
\text { Prognostic value }\end{array}$ & $\begin{array}{l}\text { Less accurate and } \\
\text { reproducible estimation of } \\
\text { LV mass compared with } \\
\text { CMR }\end{array}$ \\
\hline $\begin{array}{l}\text { LV ejection } \\
\text { fraction }\end{array}$ & $\begin{array}{l}\text { Mandatory in the decision- } \\
\text { making process } \\
\text { Established prognostic value } \\
\text { in AS }\end{array}$ & $\begin{array}{l}\text { Difficult to measure in } \\
\text { patients with suboptimal } \\
\text { acoustic window } \\
\text { Unable to detect early LV } \\
\text { dysfunction }\end{array}$ \\
\hline $\begin{array}{l}\text { Myocardial } \\
\text { velocities, } \\
\text { strain ad } \\
\text { strain rate (TDI } \\
\text { derived) }\end{array}$ & $\begin{array}{l}\text { Early markers of LV dysfunction } \\
\text { especially when assessed } \\
\text { during or after exercise in } \\
\text { asymptomatic patients with AS }\end{array}$ & $\begin{array}{l}\text { Angle dependent } \\
\text { Do not reflect global LV } \\
\text { function in patients with } \\
\text { segmental wall motion } \\
\text { abnormalities } \\
\text { Partial dependence on the } \\
\text { LV afterload }\end{array}$ \\
\hline $\begin{array}{l}\text { Global } \\
\text { longitudinal } \\
\text { strain (STE) }\end{array}$ & $\begin{array}{l}\text { Detects global LV dysfunction } \\
\text { An early marker or LV } \\
\text { decompensation } \\
\text { Prognostic value in AS }\end{array}$ & $\begin{array}{l}\text { Inter-vendor variability } \\
\text { Lack of clear thresholds to } \\
\text { determine LV dysfunction }\end{array}$ \\
\hline \multicolumn{3}{|l|}{ CMR imaging } \\
\hline LGE & $\begin{array}{l}\text { Robust observational prognostic } \\
\text { data } \\
\text { Qualitative as well as } \\
\text { quantitative assessment is of } \\
\text { value }\end{array}$ & $\begin{array}{l}\text { Access to CMR } \\
\text { Use of gadolinium contrast } \\
\text { Unable to detect early, } \\
\text { reversible interstitial fibrosis }\end{array}$ \\
\hline Native T1 & $\begin{array}{l}\text { No requirement for gadolinium } \\
\text { contrast } \\
\text { Global assessment of } \\
\text { myocardial tissue composition } \\
\text { Early assessment of reversible } \\
\text { fibrosis }\end{array}$ & $\begin{array}{l}\text { Access to CMR } \\
\text { Lack of established cut-offs } \\
\text { for normal and disease } \\
\text { states }\end{array}$ \\
\hline ECV\% & $\begin{array}{l}\text { Differentiates between } \\
\text { intracellular and extracellular } \\
\text { components of myocardium } \\
\text { Dynamic pre-AVR and } \\
\text { post-AVR } \\
\text { Early assessment of reversible } \\
\text { fibrosis }\end{array}$ & $\begin{array}{l}\text { Access to CMR } \\
\text { Use of gadolinium } \\
\text { contrast }\end{array}$ \\
\hline \multicolumn{3}{|l|}{ Cardiac CT } \\
\hline ECV & $\begin{array}{l}\text { Moderate correlation with } \\
\text { collagen volume fraction and } \\
\text { CMR ECV\% } \\
\text { Potentially no requirement for } \\
\text { blood sampling (synthetic ECV) } \\
\text { Integration into routine TAVI } \\
\text { workflow }\end{array}$ & $\begin{array}{l}\text { lodinated contrast } \\
\text { Ionising radiation }\end{array}$ \\
\hline GLS & $\begin{array}{l}\text { Good correlation with CT- } \\
\text { derived ejection fraction } \\
\text { Moderate correlation with } \\
\text { echocardiography-derived GLS } \\
\text { Potential prognostic relevance }\end{array}$ & $\begin{array}{l}\text { Iodinated contrast } \\
\text { Ionising radiation } \\
\text { Multiphase acquisition } \\
\text { Adequate image quality } \\
\text { required }\end{array}$ \\
\hline
\end{tabular}

AS, aortic stenosis; AVR, aortic valve replacement; CMR, cardiac magnetic resonance; $E C V \%$, extracellular volume fraction; GLS, global longitudinal strain; LGE, late gadolinium enhancement; LV, left ventricular; RWT, relative wall thickness; STE, speckle tracking echocardiography; TAVI, transcatheter aortic valve implantation; TDI, tissue Doppler imaging.

will be required to establish its role as a marker of subclinical LV decompensation. Moreover, careful attention should be paid to whether markers of $\mathrm{LV}$ dysfunction relate to the valve disease itself or other comorbidities, an area where CMR currently holds an advantage.

\section{Low-flow low-gradient AS with reduced LVEF}

In these patients, it is important to consider the mechanism of LVEF reduction and whether it is reversible or not. Extensive myocardial infarction or cardiomyopathy may hamper the recovery of LV function after AVR due to irreversible scar.
In a small CMR study, patients with classical low-flow lowgradient AS had significantly more replacement fibrosis and an increased burden of interstitial fibrosis compared with patients with high-gradient AS. ${ }^{\mathrm{w} 6}$

Dobutamine stress echocardiography has been widely used to identify the presence of LV flow reserve, traditionally regarded as a predictor of outcome in these patients. ${ }^{\mathrm{w} 7}$ However, although the absence of contractile reserve is related to a high operative mortality after surgical AVR, it does not predict the absence of LVEF recovery after AVR. ${ }^{\text {w8 }}$ Lack of LV contractile reserve had no effect on clinical outcomes or changes in LVEF in patients with low-flow low-gradient AS undergoing TAVI. ${ }^{\mathrm{w} 9}$ As such, the absence of flow reserve should not be considered a contraindication to AVR in patients with confirmed low-flow low-gradient severe AS. ${ }^{21}$

Longitudinal LV strain parameters were independent predictors of survival in patients with low-flow low-gradient AS, particularly in those treated conservatively. ${ }^{\mathrm{w} 10}$ Stress GLS provides significant incremental prognostic value compared with resting GLS, but its feasibility is lower.

\section{Low-flow low-gradient AS with preserved LVEF}

In this challenging setting, several parameters of LV geometry and function are used to identify patients with increased likelihood of severe AS: the degree of LVH, reduced LV longitudinal function without other explanation and low LV stroke volume. ${ }^{21}$

A reduced stroke volume can reflect a small LV cavity due to excessive LVH, with a high relative wall thickness. ${ }^{\text {w11 }}$ The decrease in stroke volume is accentuated by reduced LVEF within the normal range. ${ }^{\text {w11 }}$ In several studies, LV stroke volume was an independent predictor of survival, more powerful than LVEF. ${ }^{\mathrm{w} 12, \mathrm{w} 13}$

An association between concentric remodelling and restrictive filling was observed in these patients, probably reflecting the increased LV stiffness caused by a higher degree of myocardial fibrosis. ${ }^{\text {w11 }}$ Echocardiographic assessments of mitral annular displacement and GLS are useful to detect reduced LV longitudinal function related to fibrosis confirmed on CMR. ${ }^{23}$

Concomitant presence of severe mitral or tricuspid regurgitation, atrial fibrillation, LV dyssynchrony and right ventricular dysfunction can also contribute to low-flow states and need to be identified for optimal management. ${ }^{21}$

A prevalence of $6 \%-16 \%$ of wild-type transthyretin cardiac amyloidosis was reported in patients with severe AS. ${ }^{\text {w14,w15 }}$ Regional variations in longitudinal LV strain from base to apex with a relative 'apical sparing' pattern was suggested to differentiate cardiac amyloidosis from other causes of LVH. ${ }^{\mathrm{w} 16}$ However, in patients with severe AS, apical sparing could not predict the presence of cardiac amyloidosis, although an average tissue Doppler mitral annular S' velocity $\leq 6 \mathrm{~cm} / \mathrm{s}$ did provide a sensitive marker. ${ }^{\text {w15 }}$ Interstitial expansion associated with cardiac amyloidosis may be detected by $\mathrm{CMR},{ }^{49}$ while bone scintigraphy is the technique of choice for distinguishing transthyretin from light chain cardiac amyloidosis. ${ }^{\text {w17 }}$ Current data do not demonstrate that AVR is futile in these patients, who may still benefit from intervention and in whom novel amyloid therapies are now available. ${ }^{\mathrm{w} 18}$

The imaging techniques to interrogate myocardial function and disease discussed before, represent a potentially major advance in the assessment and management of AS. The traditional criteria for AVR are now being questioned based on our current understanding of pre-clinical myocardial disease in asymptomatic AS. Echocardiographic assessment of myocardial 


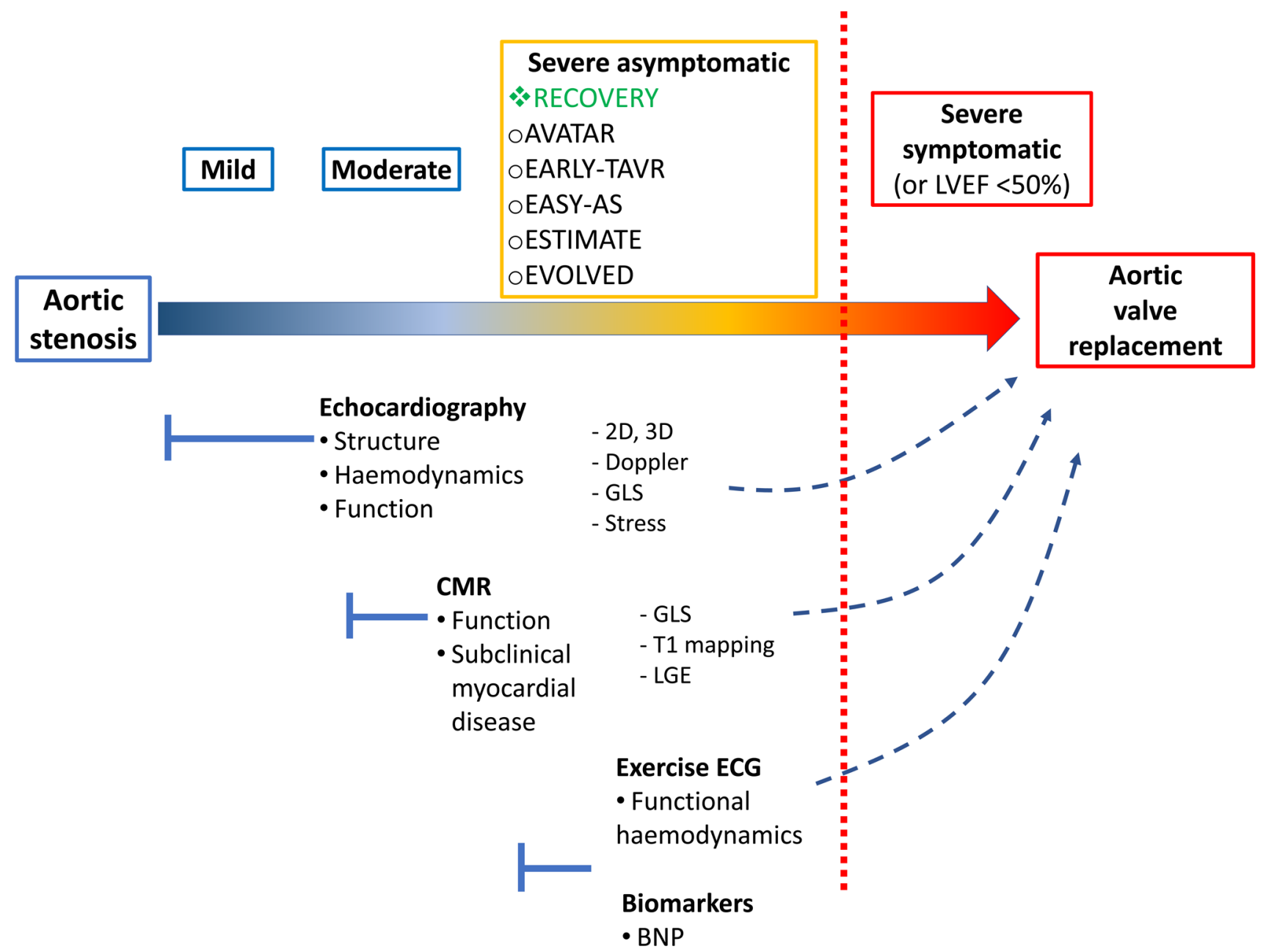

Figure 5 Proposed schematic integrating multi-modality LV assessments in AS. The current paradigm remains one of aortic valve replacement when AS is severe and symptomatic, or if there is overt evidence of impaired LV function (reduced ejection fraction). Several randomised controlled trials are assessing early intervention in severe asymptomatic AS (orange box). Of these, only RECOVERY has been completed, demonstrating a clinical benefit with early intervention in a population with very severe AS, a large proportion of which were bicuspid. EVOLVED is the only trial utilising imaging to select a subset of asymptomatic patients for early intervention. The other trials assess if early intervention could be beneficial in this setting, regardless of adjunctive risk stratification tools. Echocardiography remains, and should remain, the first-line investigation to assess AS and its effect on the LV. However, CMR adds incremental information regarding subclinical myocardial disease (fibrosis) via tissue characterisation that cannot be detected with echocardiography; these pathological changes are often present before ejection fraction falls and have prognostic implications. Other indirect assessments of LV function in response to the fixed afterload induced by AS include exercise ECG and biomarkers such as BNP. AS, aortic stenosis; BNP, B-type natriuretic peptide; CMR, cardiac magnetic resonance; RECOVERY, Randomized Comparison of Early Surgery Versus Conventional Treatment in Very Severe Aortic Stenosis, EVOLVED, Early Valve Replacement Guided by Biomarkers of LV Decompensation in Asymptomatic Patients with Severe AS; GLS, global longitudinal strain; LV, left ventricular.

deformation and CMR parameters of myocardial fibrosis offer clear prognostic information above and beyond valve haemodynamics and LVEF alone. How the use of these novel imaging techniques will improve the risk stratification of patients and optimise the timing of AVR needs to be studied in the context of randomised clinical trials (figure 5). Of note, the recent landmark RECOVERY (Randomized Comparison of Early Surgery Versus Conventional Treatment in Very Severe Aortic Stenosis) randomised controlled trial, ${ }^{\text {w19 }}$ demonstrated for the first time a mortality benefit with early surgical AVR in selected asymptomatic patients with very severe AS. The results of these important multicentre randomised controlled trials are highly anticipated and will help shape the future management paradigm of patients with AS.

References from w1 to w19 can be found in the online supplementary material.
Twitter Marc R Dweck @MarcDweck and Bogdan A Popescu @bogdan_popescu1

Contributors All authors contributed to the writing of this manuscript: conception of the review (AC, MRD and BAP), drafting of the manuscript (all authors wrote sections of the document and each section has then been edited and approved by all the other authors) and critical revision of the manuscript (all authors).

Funding MRD was supported by the British Heart Foundation (FS/14/78/31020) and is the recipient of the Sir Jules Thorn Award for Biomedical Research 2015 (15/ JTA). RB is supported by the British Heart Foundation (PG/19/40/34422).

Competing interests BAP has received research support and lecture honoraria from General Electric Healthcare.

Patient and public involvement Patients and/or the public were not involved in the design, or conduct, or reporting, or dissemination plans of this research.

Patient consent for publication Not required.

Provenance and peer review Commissioned; externally peer reviewed.

Open access This is an open access article distributed in accordance with the Creative Commons Attribution Non Commercial (CC BY-NC 4.0) license, which 
permits others to distribute, remix, adapt, build upon this work non-commercially, and license their derivative works on different terms, provided the original work is properly cited, appropriate credit is given, any changes made indicated, and the use is non-commercial. See: http://creativecommons.org/licenses/by-nc/4.0/.

\section{ORCID iD}

Bogdan A Popescu http://orcid.org/0000-0001-6122-8533

\section{REFERENCES}

1 Nkomo VT, Gardin JM, Skelton TN, et al. Burden of valvular heart diseases: a population-based study. The Lancet 2006;368:1005-11.

2 Tastet L, Tribouilloy C, Maréchaux S, et al. Staging cardiac damage in patients with asymptomatic aortic valve stenosis. J Am Coll Cardiol 2019;74:550-63.

3 Grossman W, Jones D, McLaurin LP. Wall stress and patterns of hypertrophy in the human left ventricle. J Clin Invest 1975:56:56-64.

4 Chin CWL, Everett RJ, Kwiecinski J, et al. Myocardial fibrosis and cardiac decompensation in aortic stenosis. JACC Cardiovasc Imaging 2017;10:1320-33.

5 Salcedo EE, Korzick DH, Currie PJ, et al. Determinants of left ventricular hypertrophy in patients with aortic stenosis. Cleve Clin J Med 1989;56:590-6.

6 Orlowska-Baranowska E, Placha G, Gaciong Z, et al. Influence of ACE I/D genotypes on left ventricular hypertrophy in aortic stenosis: gender-related differences. J Heart Valve Dis 2004:13:574-81

7 Briand M, Dumesnil JG, Kadem L, et al. Reduced systemic arterial compliance impacts significantly on left ventricular afterload and function in aortic stenosis: implications for diagnosis and treatment. J Am Coll Cardiol 2005;46:291-8.

8 Treibel TA, Kozor R, Fontana M, et al. Sex Dimorphism in the Myocardial Response to Aortic Stenosis. JACC Cardiovasc Imaging 2018;11:962-73.

9 Hein S, Arnon E, Kostin S, et al. Progression from compensated hypertrophy to failure in the pressure-overloaded human heart: structural deterioration and compensatory mechanisms. Circulation 2003:107:984-91.

10 Galiuto L, Lotrionte M, Crea F, et al. Impaired coronary and myocardial flow in severe aortic stenosis is associated with increased apoptosis: a transthoracic Doppler and myocardial contrast echocardiography study. Heart 2006;92:208-12.

11 Garcia D, Camici PG, Durand L-G, et al. Impairment of coronary flow reserve in aortic stenosis. J App/ Physiol 2009;106:113-21.

12 Krayenbuehl HP, Hess OM, Monrad ES, et al. Left ventricular myocardial structure in aortic valve disease before, intermediate, and late after aortic valve replacement. Circulation 1989;79:744-55.

13 Heymans S, Schroen B, Vermeersch P, et al. Increased cardiac expression of tissue inhibitor of metalloproteinase- 1 and tissue inhibitor of metalloproteinase- 2 is related to cardiac fibrosis and dysfunction in the chronic pressure-overloaded human heart. Circulation 2005; 112:1136-44.

14 Fielitz J, Hein S, Mitrovic V, et al. Activation of the cardiac renin-angiotensin system and increased myocardial collagen expression in human aortic valve disease. J Am Coll Cardiol 2001;37:1443-9.

15 Treibel TA, López B, González A, et al. Reappraising myocardial fibrosis in severe aortic stenosis: an invasive and non-invasive study in 133 patients. Eur Heart $J$ 2018:39:699-709.

16 Lang RM, Badano LP, Mor-Avi V, et al. Recommendations for cardiac chamber quantification by echocardiography in adults: an update from the American Society of echocardiography and the European association of cardiovascular imaging. Eur Heart J Cardiovasc Imaging 2015;16:233-71.

17 Takeuchi M, Nishikage T, Mor-Avi V, et al. Measurement of left ventricular mass by real-time three-dimensional echocardiography: validation against magnetic resonance and comparison with two-dimensional and m-mode measurements. J Am SoC Echocardiogr 2008;21:1001-5.

18 Gjertsson P, Caidahl K, Bech-Hanssen O. Left ventricular diastolic dysfunction late after aortic valve replacement in patients with aortic stenosis. Am J Cardiol 2005:96:722-7.

19 Sato K, Kumar A, Jones BM, et al. Reversibility of cardiac function predicts outcome after transcatheter aortic valve replacement in patients with severe aortic stenosis. J Am Heart Assoc 2017;6:pii: e005798

20 Aalaei-Andabili SH, Bavry AA. Left ventricular diastolic dysfunction and transcatheter aortic valve replacement outcomes: a review. Cardiol Ther 2019;8:21-8.

21 Baumgartner H, Falk V, Bax JJ, et al. 2017 ESC/EACTS guidelines for the management of valvular heart disease. Eur Heart J 2017:38:2739-91.

22 Ponikowski P, Voors AA, Anker SD, et al. 2016 ESC guidelines for the diagnosis and treatment of acute and chronic heart failure. Eur Heart J 2016:37:2129-200.

23 Herrmann S, Störk S, Niemann M, et al. Low-gradient aortic valve stenosis myocardial fibrosis and its influence on function and outcome. J Am Coll Cardiol 2011;58:402-12.

24 Magne J, Cosyns B, Popescu BA, et al. Distribution and Prognostic Significance of Left Ventricular Global Longitudinal Strain in Asymptomatic Significant Aortic Stenosis: An Individual Participant Data Meta-Analysis. JACC Cardiovasc Imaging 2019;12:84-92.
25 Iwahashi N, Nakatani S, Kanzaki H, et al. Acute improvement in myocardial function assessed by myocardial strain and strain rate after aortic valve replacement for aortic stenosis. J Am Soc Echocardiogr 2006; 19:1238-44.

26 Poulsen SH, Søgaard P, Nielsen-Kudsk JE, et al. Recovery of left ventricular systolic longitudinal strain after valve replacement in aortic stenosis and relation to natriuretic peptides. J Am Soc Echocardiogr 2007;20:877-84.

27 Korinek J, Wang J, Sengupta PP, et al. Two-dimensional strain--a Doppler-independent ultrasound method for quantitation of regional deformation: validation in vitro and in vivo. J Am Soc Echocardiogr 2005;18:1247-53.

28 Lafitte S, Perlant M, Reant P, et al. Impact of impaired myocardial deformations on exercise tolerance and prognosis in patients with asymptomatic aortic stenosis. Eur J Echocardiogr 2009;10:414-9.

29 Cramariuc D, Gerdts E, Davidsen ES, et al. Myocardial deformation in aortic valve stenosis: relation to left ventricular geometry. Heart 2010;96:106-12.

30 Kempny A, Diller G-P, Kaleschke G, et al. Longitudinal left ventricular 2D strain is superior to ejection fraction in predicting myocardial recovery and symptomatic improvement after aortic valve implantation. Int J Cardiol 2013;167:2239-43.

31 Lozano Granero VC, Fernández Santos S, Fernández-Golfín C, et al. Immediate improvement of left ventricular mechanics following transcatheter aortic valve replacement. Cardiol J 2018;25:487-94.

$32 \mathrm{Ng} \mathrm{ACT}$, Delgado V, Bertini M, et al. Alterations in multidirectional myocardial functions in patients with aortic stenosis and preserved ejection fraction: a twodimensional speckle tracking analysis. Eur Heart J 2011;32:1542-50.

33 Haugaa KH, Smedsrud MK, Steen T, et al. Mechanical dispersion assessed by myocardial strain in patients after myocardial infarction for risk prediction of ventricular arrhythmia. JACC Cardiovasc Imaging 2010;3:247-56.

34 Haland TF, Almaas VM, Hasselberg NE, et al. Strain echocardiography is related to fibrosis and ventricular arrhythmias in hypertrophic cardiomyopathy. Eur Heart $J$ Cardiovasc Imaging 2016;17:613-21.

35 Klaeboe LG, Haland TF, Leren IS, et al. Prognostic value of left ventricular deformation parameters in patients with severe aortic stenosis: a pilot study of the usefulness of strain echocardiography. J Am Soc Echocardiogr 2017;30:727-35.

36 Dweck MR, Joshi S, Murigu T, et al. Midwall fibrosis is an independent predictor of mortality in patients with aortic stenosis. J Am Coll Cardiol 2011;58:1271-9.

37 Barone-Rochette G, Piérard S, De Meester de Ravenstein C, et al. Prognostic significance of LGE by CMR in aortic stenosis patients undergoing valve replacement. J Am Coll Cardiol 2014:64:144-54.

38 Musa TA, Treibel TA, Vassiliou VS, et al. Myocardial scar and mortality in severe aortic stenosis. Circulation 2018:138:1935-47.

39 Balciunaite G, Skorniakov V, Rimkus A, et al. Prevalence and prognostic value of late gadolinium enhancement on CMR in aortic stenosis: meta-analysis. Eur Radiol 2020;30:640-51.

40 Everett RJ, Tastet L, Clavel M-A, et al. Progression of hypertrophy and myocardial fibrosis in aortic stenosis: a multicenter cardiac magnetic resonance study. Circ Cardiovasc Imaging 2018:11:e007451.

41 Bing R, Everett RJ, Tuck C, et al. Rationale and design of the randomized, controlled early valve replacement guided by biomarkers of left ventricular decompensation in asymptomatic patients with severe aortic stenosis (evolved) trial. Am Heart J 2019:212:91-100

42 Bing R, Cavalcante JL, Everett RJ, et al. Imaging and impact of myocardial fibrosis in aortic stenosis. JACC Cardiovasc Imaging 2019;12:283-96.

43 Puntmann VO, Peker E, Chandrashekhar Y, et al. T1 mapping in characterizing myocardial disease: a comprehensive review. Circ Res 2016;119:277-99.

44 Lee H, Park J-B, Yoon YE, et al. Noncontrast myocardial T1 mapping by cardiac magnetic resonance predicts outcome in patients with aortic stenosis. JACC Cardiovasc Imaging 2018;11:974-83.

45 Treibel TA, Bandula S, Fontana M, et al. Extracellular volume quantification by dynamic equilibrium cardiac computed tomography in cardiac amyloidosis. J Cardiovasc Comput Tomogr 2015;9:585-92.

46 Bandula S, White SK, Flett AS, et al. Measurement of myocardial extracellular volume fraction by using equilibrium contrast-enhanced CT: validation against histologic findings. Radiology 2013;269:396-403.

47 Fukui M, Xu J, Abdelkarim I, et al. Global longitudinal strain assessment by computed tomography in severe aortic stenosis patients - Feasibility using feature tracking analysis. J Cardiovasc Comput Tomogr 2019;13:157-62.

48 Fukui M, Xu J, Thoma F, et al. Baseline global longitudinal strain by computed tomography is associated with post transcatheter aortic valve replacement outcomes. J Cardiovasc Comput Tomogr 2019:30429.

49 Cavalcante JL, Rijal S, Abdelkarim I, et al. Cardiac amyloidosis is prevalent in older patients with aortic stenosis and carries worse prognosis. J Cardiovasc Magn Reson 2017;19:98.

50 Shah ASV, Chin CWL, Vassiliou V, et al. Left ventricular hypertrophy with strain and aortic stenosis. Circulation 2014;130:1607-16 